\title{
Studies of Higgs spin and parity with the ATLAS detector at the LHC
}

\section{Andrea Gabrielli on behalf of ATLAS collaboration*}

Sapienza Università di Roma and INFN Sezione di Roma 1, Piazzale Aldo Moro 2, 00185,

Roma, Italy.

E-mail: andrea.gabrielliecern.ch

Studies of the spin and parity quantum numbers of the Higgs boson candidate are presented. They are based on pp collision data collected by the ATLAS experiment at the LHC. The Standard Model spin-parity $J^{P}=0^{+}$hypothesis is confronted with alternative models using the kinematic properties of the Higgs boson decays $H \rightarrow \gamma \gamma, H \rightarrow W W^{*} \rightarrow l v l v$ and $H \rightarrow Z Z^{*} \rightarrow 4 l$. The datasets used corresponds to an integrated luminosity of $20.7 \mathrm{fb}^{-1}$ collected at $\sqrt{s}=8 \mathrm{TeV}$. For the $H \rightarrow Z Z^{*}$ channel an additional dataset corresponding to an integrated luminosity of $4.8 \mathrm{fb}^{-1}$ at $\sqrt{s}=7 \mathrm{TeV}$ is added.

The European Physical Society Conference on High Energy Physics -EPS-HEP2013 18-24 July 2013

Stockholm, Sweden

${ }^{*}$ Speaker. 
Studies of Higgs spin and parity with the ATLAS detector at the LHC Andrea Gabrielli on behalf of ATLAS collaboration

\section{Introduction}

In 2012 the ATLAS and CMS Collaborations published the discovery of a new resonance $[1,2]$ in the search for the Standard Model (SM) Higgs boson $H$. The present experimental challenge is to compare the properties of the new observed particle with the SM predictions for the Higgs boson. In the SM, the Higgs boson is a spin- 0 and CP-even particle $\left(J^{P}=0^{+}\right)[4,5]$. The LandauYang theorem forbids the direct decay of an on-shell spin-1 particle into a pair of photons. The spin-1 hypothesis is therefore strongly disfavoured by the observation of the $H \rightarrow \gamma \gamma$ decay. In this report the $J^{P}=0^{+}$hypothesis of the SM is compared to several alternative hypotheses with $J^{P}=0^{-}, 1^{+}, 1^{-}, 2^{+}$.

The measurements are based on the kinematic and angular variables of the three final states $H \rightarrow \gamma \gamma$ (cosine of the polar angle of the photons with respect to the z-axis of the Collins-Soper frame [6] is used), $H \rightarrow Z Z^{*} \rightarrow 4 l$ (multivariate discriminant based on independent angular variables and masses based on the decay planes of the 4 leptons is used, for a complete description see [7]) and $H \rightarrow W W^{*} \rightarrow l v l v$ (multivariate discriminant based on angular distribution, $p_{T}$ and masses is used [8]), where $\ell$ denotes an electron or a muon. To improve the sensitivity to different spinparity hypotheses, the final states are, in some cases, combined. To test the $J^{P}=0^{-}$spin-parity hypothesis, only the $H \rightarrow Z Z^{*} \rightarrow 4 l$ decay mode is used, while for $J^{P}=1^{+}$and $J^{P}=1^{-}$hypotheses the $H \rightarrow Z Z^{*} \rightarrow 4 l$ and $H \rightarrow W W^{*} \rightarrow l v l v$ channels are combined. For the $J^{P}=2^{+}$study, all three decay modes are combined.

\section{Results}

The results are reported in Figures 1, where the left side shows a summary of the expected and observed confidence levels for the different alternative hypotheses with respect to the SM hypothesis. For the case of the $2^{+}$hypothesis, a model corresponding to a graviton-inspired tensor with minimal couplings to SM particles ( $q \bar{q}$ fraction $f_{q \bar{q}}=4 \%$ ) is assumed (called $2_{m}^{+}$in the figure). Figure 1 (right) shows the confidence level for the alternative hypothesis as a function of the q-qbar fraction. All confidence levels are evaluated according to the CLs prescription [3].

\subsection{Test of SM $J^{P}=0^{+}$against $J^{P}=0^{-}$}

The expected and observed exclusion limits of the $J^{P}=0^{-}$hypothesis are summarised in Figure 1 (left). The data are in agreement with the $J^{P}=0^{+}$hypothesis, while the $J^{P}=0^{-}$hypothesis is excluded at $97.8 \% \mathrm{CL}$.

\subsection{Test of SM $J^{P}=0^{+}$against $J^{P}=1^{+}$}

The expected and observed exclusion limits of the $J^{P}=1^{+}$hypothesis obtained from the combination of the $H \rightarrow Z Z^{*} \rightarrow 4 l$ and $H \rightarrow W W^{*} \rightarrow l v l v$ channels is shown in Figure 1 (left). For both channels, the results are in agreement with the $J^{P}=0^{+}$hypothesis. In the $H \rightarrow Z Z^{*} \rightarrow 4 l$ channel, the $J^{P}=1^{+}$hypothesis is excluded at $99.8 \% \mathrm{CL}$, and in the $H \rightarrow W W^{*} \rightarrow l v l v$ channel, it is excluded at $92 \% \mathrm{CL}$. The combination excludes this hypothesis at $99.97 \% \mathrm{CL}$. 
Studies of Higgs spin and parity with the ATLAS detector at the LHC

\subsection{Test of SM $J^{P}=0^{+}$against $J^{P}=1^{-}$}

The expected and observed exclusion limits of the $J^{P}=1^{-}$hypothesis obtained from the combination of the $H \rightarrow Z Z^{*} \rightarrow 4 l$ and $H \rightarrow W W^{*} \rightarrow l v l v$ channels is shown in Figure 1 (left). For both channels, the results are in agreement with the $J^{P}=0^{+}$hypothesis in the $H \rightarrow Z Z^{*} \rightarrow 4 l$ channel, the $J^{P}=1^{-}$hypothesis is excluded at $94 \% \mathrm{CL}$, while in the $H \rightarrow W W^{*} \rightarrow l v l v$ channel, the $J^{P}=1^{-}$hypothesis is excluded at $98 \% \mathrm{CL}$. The combination excludes this hypothesis at $99.7 \% \mathrm{CL}$.

\subsection{Test of SM $J^{P}=0^{+}$against $J^{P}=2^{+}$}

The expected and observed exclusion limits of the $2^{+}$hypothesey in the combination of the three channels are summarised in Figures 1, for all $f_{q q}$ values of the spin-2 particle considered. For all three channels, the results are in agreement with the spin-0 hypothesis. The data are in good agreement with the Standard Model $J^{P}=0^{+}$hypothesis over the full $f_{q \bar{q}}$ range. Figure 1 (right) shows the comparison of the expected and observed CLs values for the $J^{P}=2^{+}$exclusion limitstion as a function of $f_{q \bar{q}}$. The observed exclusion of the $J^{P}=2^{+}$hypothesis in favour of the Standard Model $J^{P}=0^{+}$hypothesis exceeds $99.9 \%$ CL for all values of $f_{q \bar{q}}$.
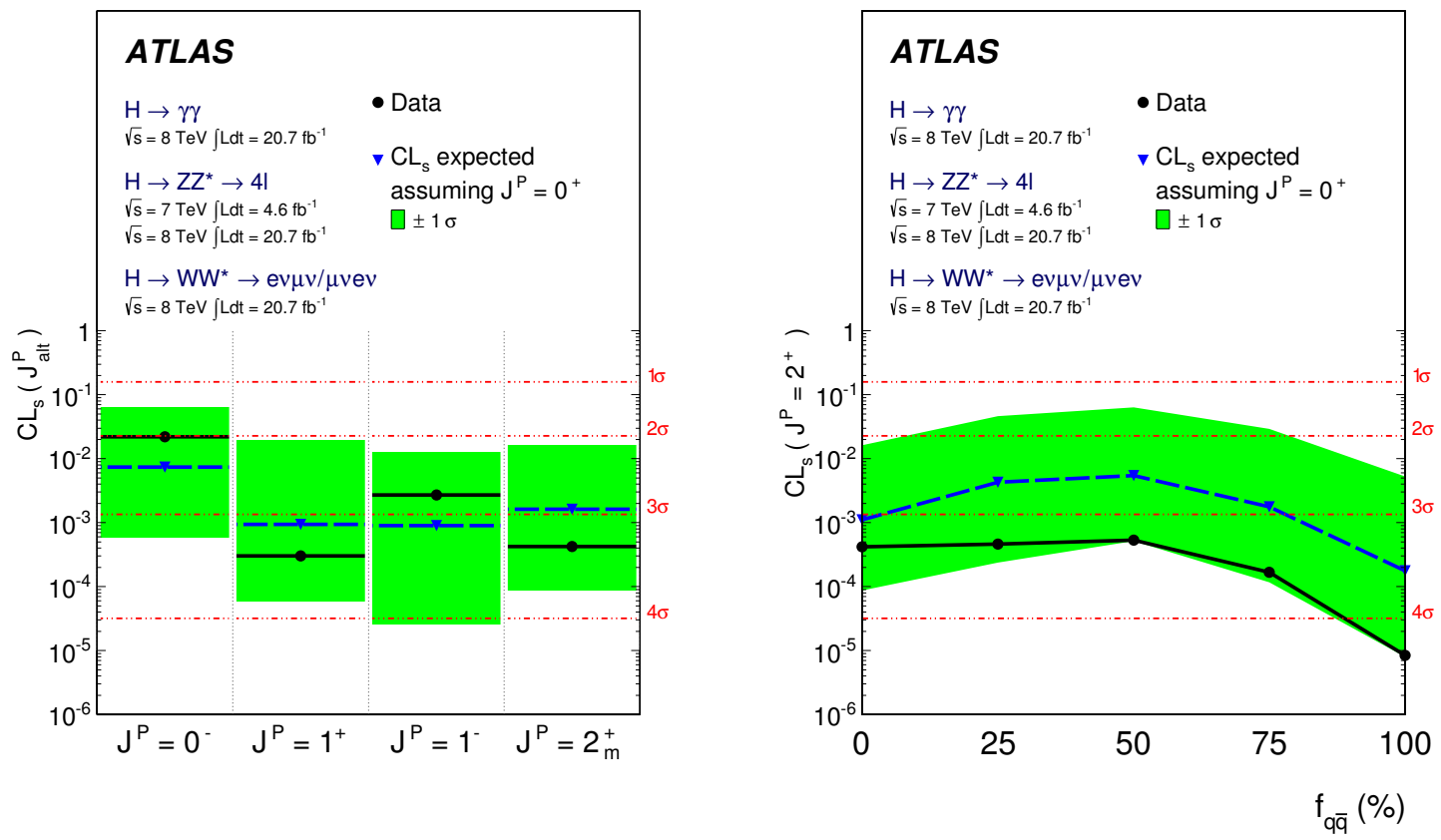

Figure 1: Expected (blue triangles/dashed lines) and observed (black circles/solid lines) confidence level for alternative spin-parity hypotheses assuming a $0^{+}$signal [4].

\section{Conclusions}

All alternative models studied are excluded without assumptions on the strength of the couplings of the Higgs boson to SM particles. These studies provide evidence for the spin-0 nature of the Higgs boson. The $0^{-}$hypothesis is rejected at $97.8 \%$ CL by using the $H \rightarrow Z Z^{*} \rightarrow 4 l$ decay alone. 
Studies of Higgs spin and parity with the ATLAS detector at the LHC

\section{References}

[1] ATLAS Collaboration, Observation of a new particle in the search for the Standard Model Higgs boson with the ATLAS detector at the LHC, Phys. Lett. B 716 (2012) 1, arXiv:1207.7214 [hep-ex].

[2] CMS Collaboration, Observation of a new boson at a mass of $125 \mathrm{GeV}$ with the CMS experiment at the LHC, Phys. Lett. B 716 (2012) 30, arXiv:1207.7235 [hep-ex].

[3] A.L. Read, J. Phys. G 28, 2693 (2002);

[4] ATLAS Collaboration, Evidence for the spin-0 nature of the Higgs boson using ATLAS data, Submitted to Physics Letters B, arXiv:1307.1432 [hep-ex].

[5] ATLAS Collaboration, Study of the spin of the new boson with up to $25 \mathrm{fb}^{-1}$ of ATLAS data, ATLAS-CONF-2013-040,

https://atlas.web.cern.ch/Atlas/GROUPS/PHYSICS/CONFNOTES/ATLAS-CONF-2013-040/.

[6] ATLAS Collaboration, Study of the spin of the Higgs-like boson in the two photon decay channel using $20.7 \mathrm{fb}^{-1}$ of pp collisions collected at $\sqrt{\mathrm{s}}=8 \mathrm{TeV}$ with the ATLAS detector, ATLAS-CONF-2013-029, https://atlas.web.cern.ch/Atlas/GROUPS/PHYSICS/CONFNOTES/ATLAS-CONF-2013-029/.

[7] ATLAS Collaboration, Measurements of the properties of the Higgs-like boson in the four lepton decay channel with the ATLAS detector using $25 \mathrm{fb}^{-1}$ of proton-proton collision data, ATLAS-CONF-2013-013, https://atlas.web.cern.ch/Atlas/GROUPS/PHYSICS/CONFNOTES/ATLAS-CONF-2013-013.

[8] ATLAS Collaboration, Study of the spin properties of the Higgs-like boson in the $H \rightarrow W W^{*} \rightarrow e v \mu v$ channel with $21 \mathrm{fb}^{-1}$ of $\sqrt{s}=8 \mathrm{TeV}$ data collected with the ATLAS detector, ATLAS-CONF-2013-031, https://atlas.web.cern.ch/Atlas/GROUPS/PHYSICS/CONFNOTES/ATLAS-CONF-2013-031. 